\title{
A NEW RADIO TELESCOPE OF HIGH RESOLVING POWER
}

\author{
S. E. KHAĬkIN AND N. L. KAİDANOVSRII \\ Academy of Sciences, Moscow, USS.R.
}

Attempts to construct large parabolic reflectors for microwaves have met with the following difficulty. For normal operation of radio telescopes, the form of the reflecting surface must not deviate from the theoretical by more than $0.1 \lambda$; therefore, the larger its size the higher the requirements for relative accuracy (i.e., the ratio of the largest permissible deviation to the cross-section of the reflector). So far, a relative accuracy better than $10^{-4}$ has not been attained in any of the existing radio telescopes, and there are no grounds for supposing that it can be increased considerably.

Since the deviation from the theoretical form of the surface should not be more than $0.1 \lambda$, then for a relative accuracy of $10^{-4}$, the diameter of the reflector $D$ cannot be much larger than $1000 \lambda$, and the beamwidth cannot be less than 3 minutes of arc (the beam angle is equal to about $\lambda / D$ at halfpower width).

For a future increase of the resolving power, large new reflectors must be constructed with a relative accuracy much higher than $10^{-4}$. A new principle, which solves the above problem, was proposed by the authors in 1952 and has been realized at the Pulkovo Observatory. The reflector consists of a large number of separate, mechanically independent, reflecting elements so adjusted that together they form, with sufficient accuracy, the necessary reflecting surface. Therefore, by enlarging the reflector (by increasing the number of reflecting elements), the higher requirement is on the relative accuracy of the relative adjustment of the elements and not on the separate elements themselves. The problem of adjusting the reflector is solved by geodetic methods, which permit an accuracy of the order of $10^{-6}$ to be attained, i.e. a much higher order than can be realized in a reflector that is a mechanically whole construction. Since the separate reflecting elements must be mounted on the ground, it is possible to increase considerably the reflector's size in a horizontal direction. The ratio telescope's directivity pattern will have a correspondingly small beam only in the horizontal direction ("fan-beam pattern"). However, as is shown in [1] and also according to the calculations of U.N. Pariiskii, the fan-beam pattern for observing one and the same object at different azimuths makes it possible in most cases to attain in both coordinates (right ascension and declination) the resolving power corresponding to a small beam angle. We can, therefore, accept the fact that the radio telescope gives a narrow directivity pattern only in the horizontal direction if observations in any azimuth can be made.

The necessary reflecting and focusing surface can be formed from the 
separate flat elements in the following manner. Imagine a paraboloid of revolution, the focus of which is fixed at a certain height above the earth's surface. For observations in different directions this "initial paraboloid" must rotate about the focus so that its axis coincides with the necessary direction (Fig. 1). The intersection of the paraboloid with the horizontal
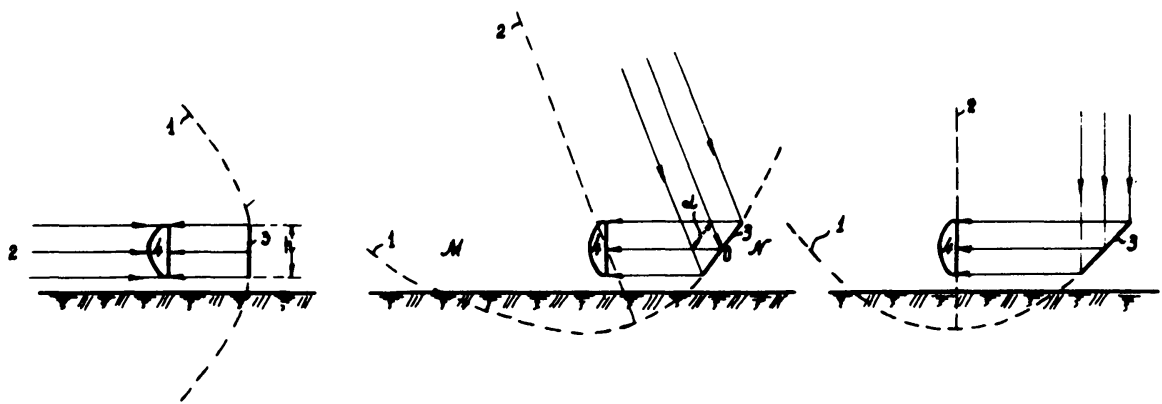

FIG. 1. (1) The imaginary paraboloid of revolution; (2) the axis of the paraboloid directed to the celestial body ; (3) the reflecting surface; (4) feed, and $\alpha=$ the altitude of the celestial body.

surface $M N$, which passes through the focus, is an ellipse (Fig. 2) defined by the equation

$$
\rho=\frac{p}{1+\cos \alpha \cos \varphi},
$$

in which $\rho$ is the distance of a point on the ellipse to the focus, $p$ is the parameter of the initial paraboloid, and $\alpha$ is the altitude of the celestial body.

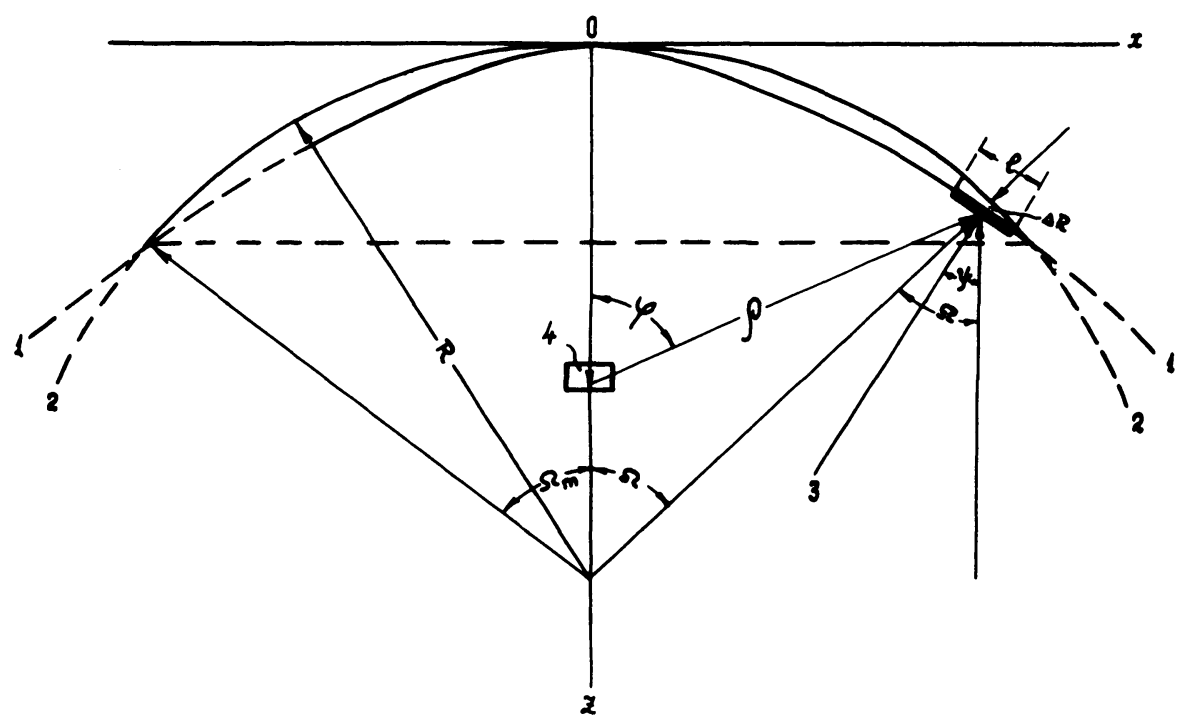

FIG. 2. (1) Ellipse; (2) circle; (3) normal ; (4) feed. 
If the flat reflecting elements are placed close to one another along this ellipse, tangent to the paraboloid, then by selecting the corresponding size of these elements, the reflecting surface will transform the plane wave along the initial paraboloidal axis into a converging cylindrical wave, which has a vertical focal line that passes through the focus of the initial paraboloid. It is necessary that the width of the element $l$ be so small $\left(l<\left(\frac{1}{2} p \lambda_{\mathrm{min}}\right)^{1 / 2}\right)$ that the path-difference from the edge of the element to the focus is small in comparison to the length of the shortest wave used, and the height $h$ so large $\left(h>\left(2 p \lambda_{\max }\right)^{1 / 2} \sec \frac{1}{2} \alpha\right)$ that the diffraction of the longest wave used is negligible in propagation between the reflecting elements and the focus. Then the rays, reflected from all the points of the element that lie in the plane $M N$, will come to the focus of the initial paraboloid in one and the same phase. The rays reflected from points of the element that lie in the same vertical plane will come in the same phase to points at respective heights on the vertical line passing through the focus (Fig. 1). This means that the surface formed by the reflecting elements will transform the plane wave into a cylindrical wave that spreads in a horizontal direction and converges at a focal line passing through the paraboloid's focus. By placing near the focus a second reflector in the form of a parabolic cylinder, with a horizontal generatrix and a focus situated symmetrically to the main reflector's focus, it is possible to transform the converging cylindrical wave into a spherical wave that converges on the phase center of a feed of any type.

Because the many-sided reflector has periodic errors and slits between the separate reflecting elements, far and weak diffracting lobes appear and, therefore, the area efficiency coefficient decreases (by several per cent) but the form of the major lobe is not influenced.

Since for all angles of observation the focus of the initial paraboloid is always in one horizontal plane (near the earth's surface), it is necessary to adjust the feed in a given direction by moving it only in a horizontal plane and by rotating it about the vertical axis.

The antenna's reflecting surface is given the necessary form by a translational movement of the reflecting elements and also by a rotation about the . horizontal and vertical axes.

The translational movements can be reproduced with sufficient accuracy by moving the reflecting element only along the radius of the circle on which the centers of these elements should be fixed when observing in the zenith. The relative movement of the reflecting elements in the radial direction is given by:

$$
\begin{aligned}
\delta & =\frac{\Delta R}{R} \\
& =\frac{\cos \Omega\left(\sin ^{2} \alpha-q\right)+\sqrt{\cos ^{2} \Omega\left(\sin ^{2} \alpha-q\right)^{2}+\left(2 q-\sin ^{2} \alpha\right)\left(1-\cos ^{2} \alpha \cos ^{2} \Omega\right.}}{1-\cos ^{2} \alpha \cos ^{2} \Omega}-1,
\end{aligned}
$$

in which $\Omega$ is the angle of the reflecting elements as seen from the center of the circle (Fig. 2), $q=p / R$. 
From the point of view of construction, it is essential that the radial movement be small. This is accomplished by selecting for each angle $\alpha$ the most suitable parameter $p$ of the initial paraboloid, for which the curve of the middle line of the reflecting surface differs minimally from a circle of radius $R$.

The angle of inclination $\beta$ of the reflecting element, seen from the focus under the angle $\varphi$ can be determined from:

$$
\sin \beta=\frac{\sin \alpha}{\sqrt{2(1+\cos \varphi \cos \alpha)}} .
$$

The reflecting elements need to be rotated about the vertical axis through the angle $\Omega-\psi$, in which $\psi$ is determined by

$$
\tan \psi=\frac{(1-\delta) \sin \Omega}{\sqrt{q^{2}-(1+\delta)^{2} \sin ^{2} \Omega \sin ^{2} \alpha}} .
$$

By mounting the reflecting elements along the whole circumference we get the possibility of observing in all azimuths. If there are automatic operating devices, a rapid adjustment of all the reflecting elements for observations in the necessary direction may be made, and the scheduled program of following the source can be observed. The above principle has been partly realized in the large radio telescope at the Pulkovo Observatory (Fig. 3). At present the radio telescope has 90 reflecting elements of dimensions $l=1.5 \mathrm{~m}$,

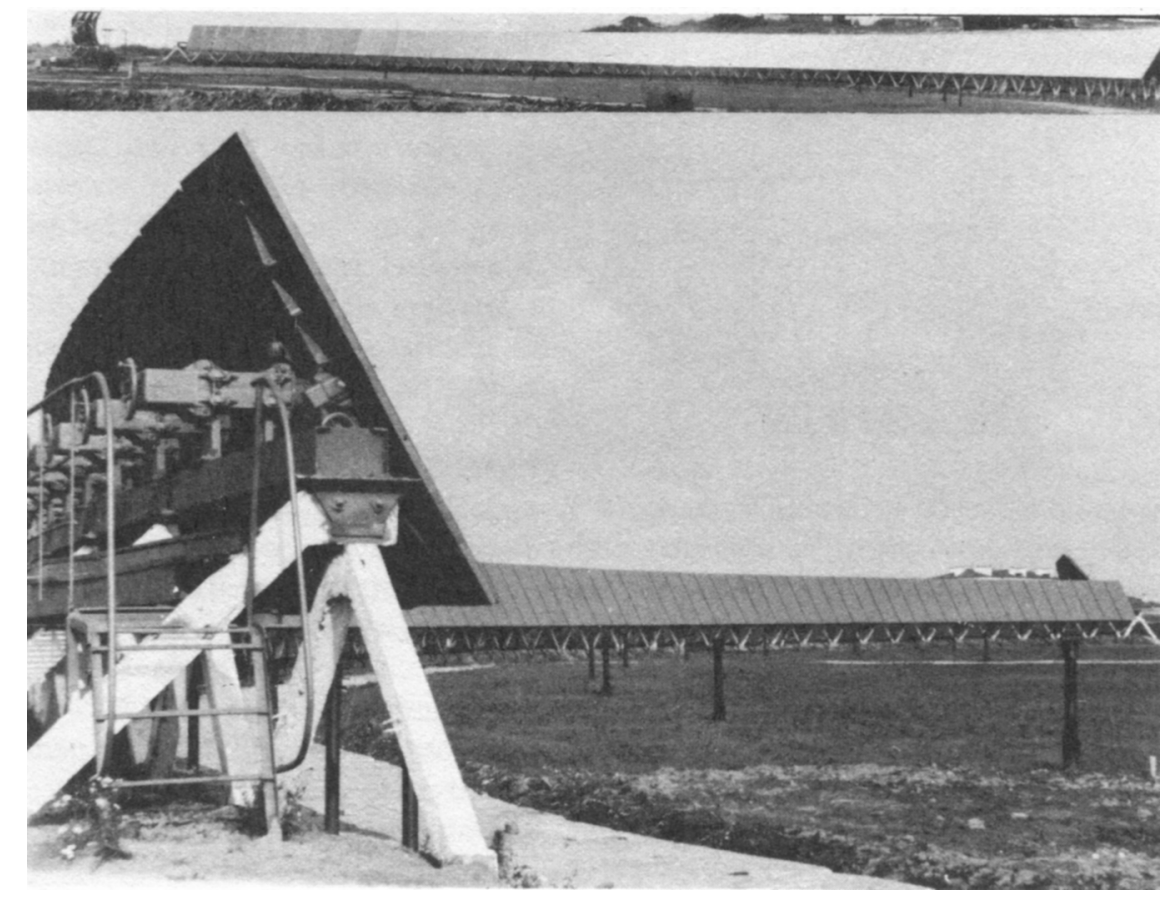

FIG. 3. General views of the Pulkovo radio telescope. 
$h=3 \mathrm{~m}$, placed along an arc $2 \Omega_{m} \approx 90$ degrees of the circle with a radius $R=100 \mathrm{~m}$. Each reflecting element has a device that permits a movement in the radial direction of $30 \mathrm{~cm}$ and also a rotation on the horizontal and vertical axes. The reflecting elements are adjusted by hand according to the scales attached to the mechanisms and spirit levels.

The completed radio telescope at wavelength $3.2 \mathrm{~cm}$ has a fan-beam diagram one minute of arc in width and from 10 minutes to one degree in height (depending on the altitude of the direction of observation).

The principle proposed allows the construction of a microwave radio telescope with an area of about twenty thousand square meters. This principle can also be used with success at longer wavelengths. It is possible to construct a radio telescope of area $100,000 \mathrm{~m}^{2}$ for a range of wavelengths from $20 \mathrm{~cm}$ to $2 \mathrm{~m}$.

REFERENCE

[1] Bracewell, R. N. Aust. J. Phys. 9, 297, 1956. 